\title{
Cord Blood Alkaline Phosphatase as an Indicator of Neonatal Jaundice
}

\author{
Mousa Ahmadpour-Kacho, ${ }^{1}$ Yadollah Zahed Pasha, ${ }^{1}$ Mohsen Haghshenas, ${ }^{1}$ Zahra Akbarian \\ Rad, ${ }^{1}$ Alireza Firouzjahi, ${ }^{1}$ Ali Bijani, ${ }^{1}$ Abdollah Dehvari, ${ }^{2, *}$ and Mehrangiz Baleghi ${ }^{1}$ \\ ${ }^{1}$ Babol University of Medical Sciences, Babol, IR Iran \\ ${ }^{2}$ Children And Adolescent Health Research Center, Zahedan University of Medical Sciences, Zahedan, IR Iran \\ *Corresponding author: Abdollah Dehvari, Children And Adolescent Health Research Center, Zahedan University of Medical Sciences, Zahedan, IR Iran. E-mail: dehvari.abdh@yahoo.com
}

Received: February 16, 2015; Accepted: July 29, 2015

\begin{abstract}
Background: Management of hyperbilirubinemia remains a challenge for neonatal medicine because of the risk of neurological complications related to the toxicity of severe hyperbilirubinemia.

Objectives: The purpose of this study was to examine the validity of cord blood alkaline phosphatase level for predicting neonatal hyperbilirubinemia.

Patients and Methods: Between October and December 2013 a total of 102 healthy term infants born to healthy mothers were studied. Cord blood samples were collected for measurement of alkaline Phosphatase levels immediately after birth. Neonates were followed-up for the emergence of jaundice. Newborns with clinical jaundice were recalled and serum bilirubin levels measured. Appropriate treatment based on serum bilirubin level was performed. Alkaline phosphatase levels between the non-jaundiced and jaundiced treated neonates were compared.

Results: The incidence of severe jaundice that required treatment among followed-up neonates was 9.8\%. The mean alkaline phosphatase level was 309.09 $\pm 82.51 \mathrm{IU} / \mathrm{L}$ in the non-jaundiced group and $367.80 \pm 73.82 \mathrm{IU} / \mathrm{L}$ in the severely jaundiced group $(\mathrm{P}=0.040)$. The cutoff value of $314 \mathrm{IU} / \mathrm{L}$ was associated with sensitivity $80 \%$ and specificity $63 \%$ for predicting neonatal hyperbilirubinemia requiring treatment

Conclusions: The cord blood alkaline phosphatase level can be used as a predictor of severe neonatal jaundice.
\end{abstract}

Keywords: Fetal Blood; Hyperbilirubinemia; Jaundice; Alkaline Phosphatese; Newborn

\section{Background}

Due to early discharge of infants from the hospital, readmission has been increased (1). Therefore, early diagnosis of jaundice and timely actions are necessary. Several methods have been used to determine risk of neonatal hyperbilirubinemia. The measurement of bilirubin level (2) and alpha fetoprotein (3) in cord blood have been used for this purpose. For the first time Aysin Nalbantoglu et al. used alkaline phosphatase (ALP) level 6 hours after birth as a marker for determining hemolysis and hyperbilirubinemia (4). Alkaline phosphatase is a hydrolase enzyme and responsible for removing phosphate from many types of molecules (5). Alkaline phosphatase is found in almost all body cells, including red blood cells (4-6).

\section{Objectives}

Therefore, we hypothesized that it can be used as a marker for early diagnosis of hyperbilirubinemia. This study was conducted to evaluate the ALP level as a marker for early diagnosis of neonatal jaundice.

\section{Patients and Methods}

In this prospective study, between October and December 2013, infants who were born at the Babol-Clinic Hospital in Babol, Northern Iran, were selected. A total of 105 healthy term infants with gestational age between 37 and 42 weeks, weighing more than $2500 \mathrm{~g}$ born to healthy mothers were studied. Five milliliter cord blood was taken after birth and sent for determination of ALP level. Serum alkaline phosphatase was measured with auto analyzer (Sinnowa-DS301, China, 2013). Infants who were born to mothers with diseases such as eclampsia, diabetes, bone, kidney and liver diseases, and infants who were found suffering from other diseases except jaundice were excluded from the study. Three cases were lost to the study, so 102 cases were monitored for the emergence of clinical jaundice based on clinical observation by parents or physicians up to 10 days after birth. Infants with clinical jaundice were recalled and serum bilirubin level was measured and treated based on American Academy of Pediatrics (AAP) protocols. These were assigned as the treatment group and neonates without clinical jaundice were assigned as the non-jaundiced group. In treatment group, to deter-

Copyright (C) 2015, Growth \& Development Research Center. This is an open-access article distributed under the terms of the Creative Commons Attribution-NonCommercial 4.0 International License (http://creativecommons.org/licenses/by-nc/4.0/) which permits copy and redistribute the material just in noncommercial usages, provided the original work is properly cited. 
mine the cause of hyperbilirubinemia, work-up including complete blood count, reticulocyte count, estimation of blood group in mother and neonate, peripheral blood smear, evaluation of G6PD level and Coombs test, was done. Demographic information of all infants including gestational age, birth weight and Apgar score was recorded. The results were analyzed by SPSS 22 software.

\section{Results}

A total of 105 cases were followed-up. Three cases were lost to the study. The remaining 102 cases consisted of 50 (49\%) males and 52 (51\%) females. Ninety eight (96\%) infants were born by cesarean section and 4 (4\%) by vaginal delivery. Apgar scores were normal (9-10) at birth in all cases. The mean gestational age was 38.7 weeks and the mean birth weight 3649.59 grams (Table 1). The incidence of clinical jaundice during follow-up was $47 \%$. In $39.2 \%$ bilirubin reached a peak of $\leq 10 \mathrm{mg} / \mathrm{dL}$. The rate of need for treatment was $9.8 \%$ (10 cases), of which 5 cases were ABO incompatible, one case Rh incompatible, 2 cases G6PD deficient and in 2 cases the cause of jaundice remained unknown. Hct levels and reticulocyte count were in normal range and Coombs test was negative in these cases. None of the neonates needed exchange transfusion.

There was no difference between groups with regard to gestational age, birth weight and Apgar scores, but the comparison of cord blood alkaline phosphatase levels revealed a significant difference between the two groups ( $P$ value $=0.041)($ Table 2$)$.

Comparison of cord blood alkaline phosphatase levels between non-jaundiced group and jaundiced newborns in whom bilirubin level had reached $\leq 10 \mathrm{mg} / \mathrm{dL}$, revealed a significant difference $(P$ value $=0.016)($ Table 3$)$.

Comparison of the non-jaundiced group with neonates who required treatment according to AAP protocol (the treatment group) showed a significant difference in cord blood alkaline phosphatase levels $($ Pvalue $=0.040)($ Table 4$)$.

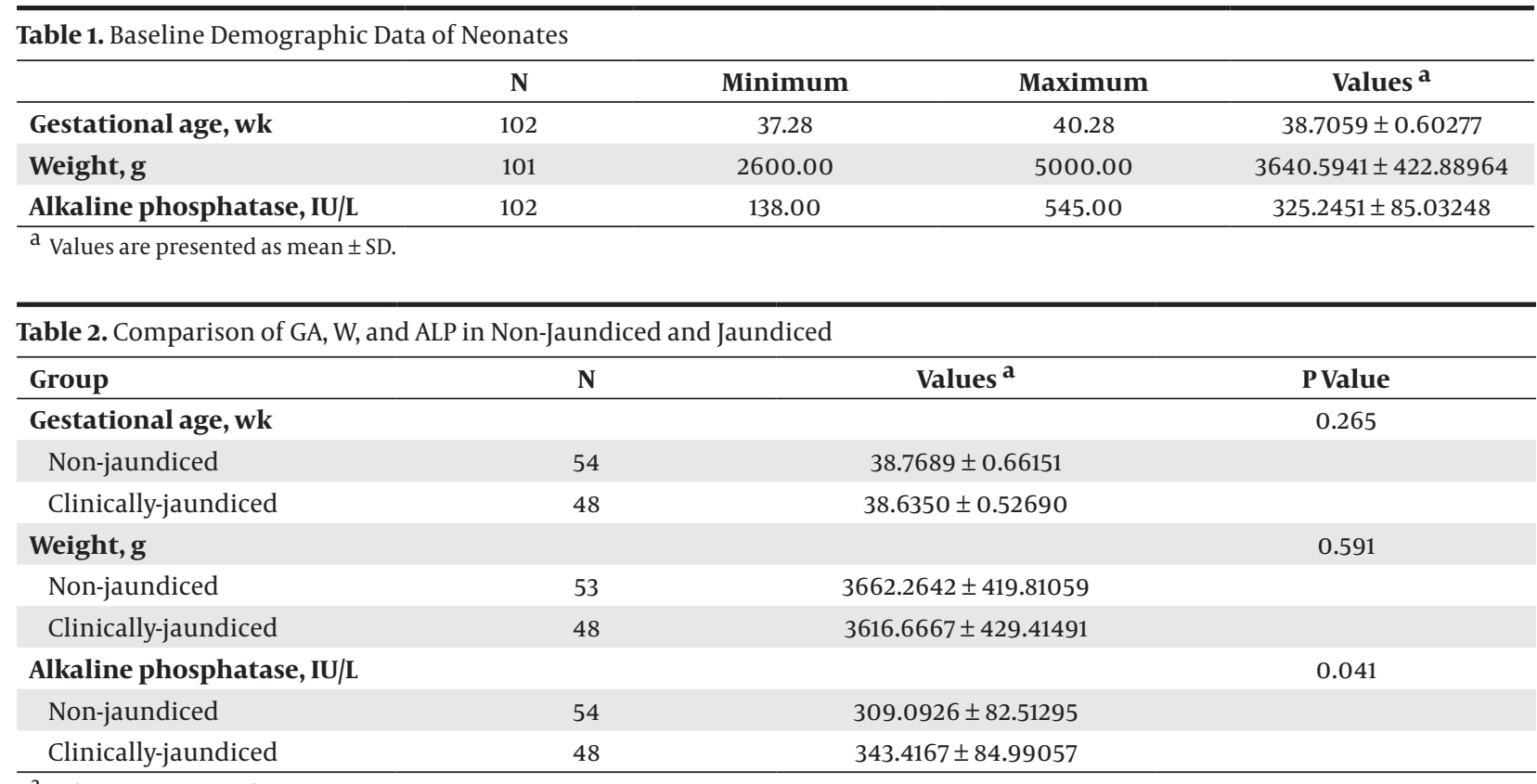

a Values are presented as mean \pm SD.

\begin{tabular}{|c|c|c|c|}
\hline Group & $\mathbf{N}$ & Values $^{a}$ & P Value \\
\hline Gestational age, wk & & & 0.139 \\
\hline Non-jaundiced & 54 & $38.7689 \pm 0.66151$ & \\
\hline $\mathrm{TSB} \geq 10, \mathrm{mg} / \mathrm{dL}$ & 40 & $38.5770 \pm 0.55053$ & \\
\hline Weight, g & & & 0.415 \\
\hline Non-jaundiced & 53 & $3662.2642 \pm 419.81059$ & \\
\hline $\mathrm{TSB} \geq 10 \mathrm{mg} / \mathrm{dL}$ & 40 & $3592.5000 \pm 388.54594$ & \\
\hline Alkaline phosphatase, IU/L & & & 0.016 \\
\hline Non-jaundiced & 54 & $309.0926 \pm 82.51295$ & \\
\hline $\mathrm{TSB} \geq 10 \mathrm{mg} / \mathrm{dL}$ & 40 & $352.6000 \pm 88.64241$ & \\
\hline
\end{tabular}

\footnotetext{
a Values are presented as mean \pm SD.
} 
Ahmadpour-Kacho M et al.

\begin{tabular}{|c|c|c|c|}
\hline Group & $\mathbf{N}$ & Values $^{a}$ & P Value \\
\hline Gestational age, wk & & & 0.473 \\
\hline Non-jaundiced & 54 & $38.7689 \pm 0.66151$ & \\
\hline Treatment & 10 & $38.6080 \pm 0.55707$ & \\
\hline Weight, $\mathrm{g}$ & & & 0.745 \\
\hline Non-jaundiced & 53 & $3662.2642 \pm 419.81059$ & \\
\hline Treatment & 10 & $3710.0000 \pm 443.34586$ & \\
\hline Alkaline phosphatase, IU/L & & & 0.040 \\
\hline Non-jaundiced & 54 & $309.0926 \pm 82.51295$ & \\
\hline Treatment & 10 & $367.8000 \pm 73.82231$ & \\
\hline
\end{tabular}

a values are presented as mean \pm SD.

Figure 1. Receiver Operating Characteristic (ROC) Curve of Cord Blood Alkaline Phosphatase

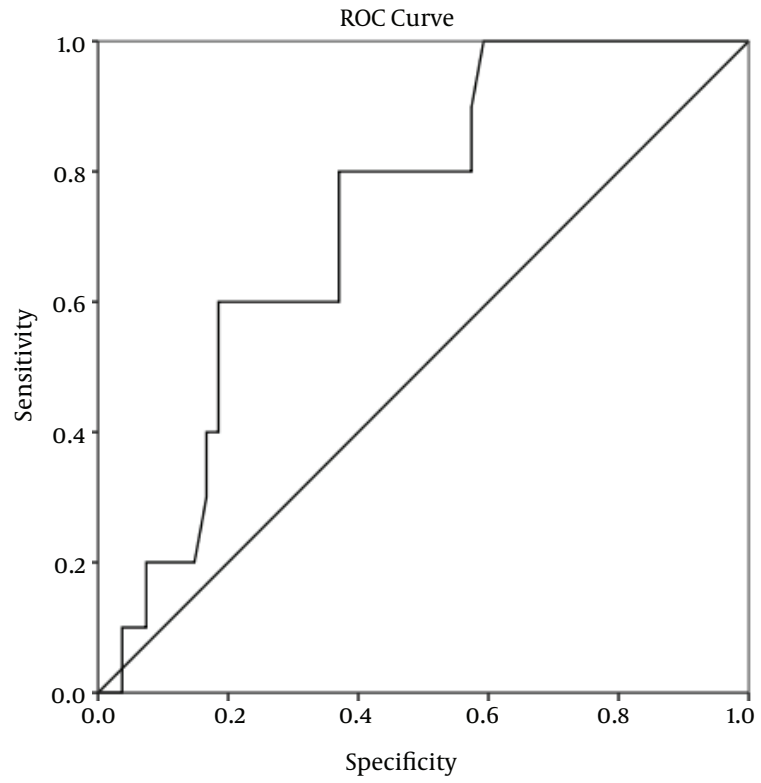

Diagonal segments are produced by ties.

(ALP) at the treatment group, area under the curve is 0.730 .

A comparison of the ROC curves of the alkaline phosphatase levels between the non-jaundiced and treatment groups (Figure 1) revealed that a cord blood alkaline phosphatase level > 314 IU/L was the most suitable cutoff value for predicting severe jaundice (that needs treatment). This cut-off value was associated with $80 \%$ sensitivity and $63 \%$ specificity.

Of 60 neonates whose cord blood alkaline phosphatase measured less than $314 \mathrm{IU} / \mathrm{L}$, only two neonates needed treatment. Thus, the negative predictive value of cord blood alkaline phosphatase for occurrence of hyperbilirubinemia was $96.6 \%$.

\section{Discussion}

To our knowledge this is the first clinical study that examines cord blood alkaline phosphatase level as an indicator to predict severe neonatal jaundice. The results of our study indicate that measurement of cord blood alkaline phosphatase may be a predicting marker for neonatal jaundice that can exceed $10 \mathrm{mg} / \mathrm{dL}$ and necessitates treatment in the first week of life. Cord blood alkaline phosphatase level with sensitivity and specificity of $80 \%$ and $63 \%$ respectively in cutoff level $>314 \mathrm{IU} / \mathrm{L}$ predicts a need for treatment.

Nalbantoglu et al. used blood alkaline phosphatase levels 6 hours after birth. They found that ALP levels were significantly higher in patients with hyperbilirubinemia requiring treatment, either with phototherapy or exchange transfusion (P value 0.0001) (4). In our study, there was a significant difference in the levels of cord blood alkaline phosphatase between the non-jaundiced and clinically jaundiced newborns, and it was significantly higher in patients with hyperbilirubinemia requiring treatment. Moreover, the ALP levels were significantly higher in newborns whose serum bilirubin level reached a level $\geq 10 \mathrm{mg} / \mathrm{dL}$. These findings confirm the results of Nalbantoglu et al. (4). One of advantages in our study was the site of sample collection, which was taken from cord blood. Cord blood sample predicts hyperbilirubinemia earlier than a sample taken after birth does. In addition, the neonate may not be lost to follow-up because of early discharge.

Chou et al. measured cord blood hydrogen peroxide level for prediction of neonatal hyperbilirubinemia. The cord blood hydrogen peroxide signal level of 2500 counts/10 seconds was an appropriate cutoff for predicting severe hyperbilirubinemia with sensitivity and negative predictive value of $76.2 \%$ and $93.3 \%$, respectively (7). Our study showed that the alkaline phosphatase level of $314 \mathrm{IU} / \mathrm{L}$ was associated with sensitivity and negative predictive value of $80 \%$ and $96.6 \%$, respectively.

The rate of need for treatment of jaundice in our study was $9.8 \%$ (10 cases). Of these, 5 cases were ABO incompat- 
ible, one Rh incompatible, 2 G6PD deficient and 2 cases were of unknown etiology. According to these findings, it seems that the alkaline phosphatase level has a higher validity in disclosing hemolytic processes.

Various tests have been studied to predict hyperbilirubinemia. Knupfer et al. (8) used the cord blood bilirubin level to predict the need for phototherapy. By a cord bilirubin cut-off level of $30 \mu \mathrm{mol} / \mathrm{L}$ this revealed a sensitivity of $70.3 \%$ and a negative predictive value of $65.6 \%$. Our study had a sensitivity and negative predictive value of $80 \%$ and $96.6 \%$, respectively. The measurement of end tidal carbon monoxide (9) at the cutoff level of $1.8 \mu / \mathrm{L}$ (ppm) showed a negative predictive value $97 \%$; our study showed a negative predictive value of $96.6 \%$, which is comparable to this expensive and low accessible test. The first day serum bilirubin (10) and sixth hour serum bilirubin (11) have been used for prediction of hyperbilirubinemia. These tests require venous blood sampling (in many countries as in ours), and so are not suitable for screening all neonates.

In our study the mean level of alkaline phosphatase in the studied newborns was $325.24 \pm 85.03 \mathrm{IU} / \mathrm{L}$, which is more than that in the existing reports. Fenton et al. found the mean level of cord blood alkaline phosphatase $159 \pm 49 \mathrm{IU} / \mathrm{L}(12)$. Compared with our results, there is a big difference. Another local study by Abbasian et al. in Shahrood, Iran showed that mean cord blood alkaline phosphatase level was $314.34 \pm 122.42 \mathrm{IU} / \mathrm{L}$, which is compatible with our findings (13). The average level of cord blood alkaline phosphatase in Iranian newborns seems to be higher than in other populations.

Jaundiced newborns had higher cord blood alkaline phosphatase levels than non-jaundiced newborns. Cord blood alkaline phosphatase level is a useful indicator in predicting subsequent jaundice in healthy term newborns.

\section{Acknowledgements}

We would like to thank Clinical Research Development Committee of Amirkola Children's Hospital and Dr. AR
Beigi and all the laboratory personnel for their kind and valuable assistance.

\section{References}

1. Sgro M, Campbell D, Shah V. Incidence and causes of severe neonatal hyperbilirubinemia in Canada. CMAJ. 2006;175(6):587-90.

2. Zeitoun AA, Elhagrasy HF, Abdelsatar DM. Predictive value of umbilical cord blood bilirubin in neonatal hyperbilirubinemia. Gaz Egypt Paediatr Assoc. 2013;61(1):23-30.

3. Tan KL, Loganath A, Roy AC, Goh HH, Karim SM, Ratnam SS. Cord plasma alpha-fetoprotein values and neonatal jaundice. Pediatrics. 1984;74(6):1065-8.

4. Nalbantoglu A, Ovali F, Nalbantoglu B. Alkaline phosphatase as an early marker of hemolysis in newborns. Pediatr Int. 2011;53(6):936-8.

5. Tinnion RJ, Embleton ND. How to use... alkaline phosphatase in neonatology. Arch Dis Child Educ Pract Ed. 2012;97(4):157-63.

6. Monanu MO, Uwakwe AA, Onwubiko D. In vitro effects of sodium benzoate on the activities of aspartate and alanine amino transferases, and alkaline phosphatase from human erythrocytes of different genotypes. Biokemistri. 2005;17(1):33-8.

7. Chou HC, Chien CT, Tsao PN, Hsieh WS, Chen CY, Chang MH. Prediction of severe neonatal hyperbilirubinemia using cord blood hydrogen peroxide: a prospective study. PLoS One. 2014;9(1):e86797.

8. Knupfer M, Pulzer F, Gebauer C, Robel-Tillig E, Vogtmann C. Predictive value of umbilical cord blood bilirubin for postnatal hyperbilirubinaemia. Acta Paediatr. 2005;94(5):581-7.

9. Okuyama H, Yonetani M, Uetani Y, Nakamura H. End-tidal carbon monoxide is predictive for neonatal non-hemolytic hyperbilirubinemia. Pediatr Int. 2001;43(4):329-33.

10. Alpay F, Sarici SU, Tosuncuk HD, Serdar MA, Inanc N, Gokcay E. The value of first-day bilirubin measurement in predicting the development of significant hyperbilirubinemia in healthy term newborns. Pediatrics. 2000;106(2):E16.

11. Sarici SU, Yurdakok M, Serdar MA, Oran O, Erdem G, Tekinalp G et al. An early (sixth-hour) serum bilirubin measurement is useful in predicting the development of significant hyperbilirubinemia and severe $\mathrm{ABO}$ hemolytic disease in a selective high-risk population of newborns with $\mathrm{ABO}$ incompatibility. Pediatrics. 2002;109(4):e53.

12. Fenton TR, Lyon AW, Rose MS. Cord blood calcium, phosphate, magnesium, and alkaline phosphatase gestational agespecific reference intervals for preterm infants. BMC Pediatr. 2011;11(1):76.

13. Abbasian M, Chaman R, Delvarian Zadeh M, Amiri M, Raei $\mathrm{M}$, Norouzi $\mathrm{P}$, et al. Investigating the prevalence of calcium deficiency and some of its influencing factors in pregnant women and their neonates [in Persian]. Knowledge Health . 2012;7(1):39-43. 